\title{
Design Criteria and Optical Characteristics of One-Dimensional Photonic Crystals Based on Periodically Grooved Silicon
}

\author{
Vladimir Tolmachev \\ Ioffe Physico-Technical Institute \\ Tatiana Perova \\ Trinity College Dublin \\ Kevin Berwick \\ Technological University Dublin, kevin.berwick@tudublin.ie
}

Follow this and additional works at: https://arrow.tudublin.ie/engscheceart

Part of the Electrical and Electronics Commons

\section{Recommended Citation}

Tolmachev, V., Perova, T., Berwick, K.:Design Criteria and Optical Characteristics of One-Dimensional Photonic Crystals Based on Periodically Grooved Silicon. Applied Optics, Vol. 42, Issue 28, pp. 5679-5683 (2003) http://dx.doi.org/10.1364/A0.42.005679

This Article is brought to you for free and open access by the School of Electrical and Electronic Engineering at ARROW@TU Dublin. It has been accepted for inclusion in Articles by an authorized administrator of ARROW@TU Dublin. For more information, please contact arrow.admin@tudublin.ie, aisling.coyne@tudublin.ie, gerard.connolly@tudublin.ie.

Funder: Enterprise Ireland International Programme, the European Commission and the Russian government

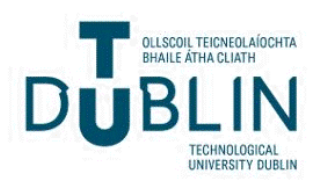




\title{
Design criteria and optical characteristics of one-dimensional photonic crystals based on periodically grooved silicon
}

\author{
Vladimir Tolmachev, Tatiana Perova, and Kevin Berwick
}

\begin{abstract}
Photonic bandgap (PBG) regions have been calculated for periodically grooved Si structures, acting as a one-dimensional photonic crystal. The wavelength range of the PBG as a function of the ratio $\left(D_{\mathrm{Si}} / A\right)$ is presented, where $D_{\mathrm{Si}}$ is the width of the $\mathrm{Si}$ walls and $A$ is the grooved silicon lattice constant. The influence of the parameter $D_{\mathrm{Si}}$, the refractive index of the space between the Si walls and the number of structure periods, $m$, on the forming of PBG regions is discussed. A good correlation between the calculated and the experimentally observed PBG regions is obtained. (C) 2003 Optical Society of America OCIS codes: $300.6340,310.3840,160.6000$.
\end{abstract}

\section{Introduction}

One-dimensional (1D) photonic crystals (PCs) with a regular change in the refractive index $n$, can be easily fabricated with the well-known technique of multilayer coating onto different substrates. ${ }^{1}$ With this technique, structures with refractive-index changes in the vertical direction can be obtained. These types of structures can be utilized for the manipulation of optical beams propagating perpendicular to the substrate plane. It is well known that $1 \mathrm{D} \mathrm{PCs}$ can, under certain conditions, exhibit properties similar to three-dimensional PCs. For example, they can show complete reflection of incident light of any polarization and all angles over a wide spectral range; that is, they can act as omnidirectional reflectors. $^{2,3}$ However, these reflectors are designed for a light beam that propagates in the vertical direction with respect to the substrate, since the refractive index is modulated in the vertical direction only. To implement the 1D PC based on Si using current fab-

V. Tolmachev is with Ioffe Physico-Technical Institute, Polytechnicheskaya 26, St. Petersburg, Russia. T. Perova (perovat@tcd.ie) is with the Department of Electronic and Electrical Engineering, University of Dublin, Trinity College, Dublin 2, Ireland. K. Berwick is with the Department of Electronic and Communications Engineering, Dublin Institute of Technology, Kevin Street, Dublin 8, Ireland.

Received 13 March 2003; revised manuscript received 23 June 2003.

0003-6935/03/285679-05\$15.00/0

(C) 2003 Optical Society of America rication technologies, it is necessary to mould the light flow in the horizontal direction.

It has been recently shown ${ }^{4-6}$ that it is possible to fabricate horizontally aligned periodic structures on Si. Foresi et al..$^{4}$ and Villeneuve et al..$^{5}$ describe a $1 \mathrm{D}$ $\mathrm{PC}$ structure consisting of a Si strip containing a periodic array of holes, resulting in a PBG region between 1.3 and $1.7 \mu \mathrm{m}$. Tolmachev et al. ${ }^{6}$ fabricated a periodic array of grooves in a (110) Si substrate and proposed its use as a horizontally aligned 1D PC. The grooved silicon structure is fabricated by wet anisotropic etching of (110) $\mathrm{Si}$ in concentrated $\mathrm{KOH}: \mathrm{H}_{2} \mathrm{O}$ solution. Photolithography is used to pattern a periodic array onto the resist. This resist pattern is then used to vertically etch (110) Si via a $\mathrm{SiO}_{2}$ mask, resulting in a periodic structure of alternating Si walls and air. ${ }^{7}$ As a result of this etching process, vertical grooves with uniform and highquality smooth Si walls up to $200 \mu \mathrm{m}$ high can be obtained (Fig. 1). Although Kendall 7 in 1979 suggested the use of these structures as far-IR filters, to our knowledge their optical properties have not been reported to date.

The possibility of fabricating 1D PCs, based on grooved $\mathrm{Si}$ and the measurement of their reflection spectra $R$ in the IR range $(\lambda=2-15 \mu \mathrm{m})$, has been reported. ${ }^{8,9}$ Tolmachev et al.8,9 have measured the reflection spectra of a number of periodically grooved Si samples using an FTS 6000 FTIR spectrometer in conjunction with a UMA 500 microscope, together with a specially designed attachment that allows the positioning of the sample in three directions. This setup allows the measurement of Si structures with a 


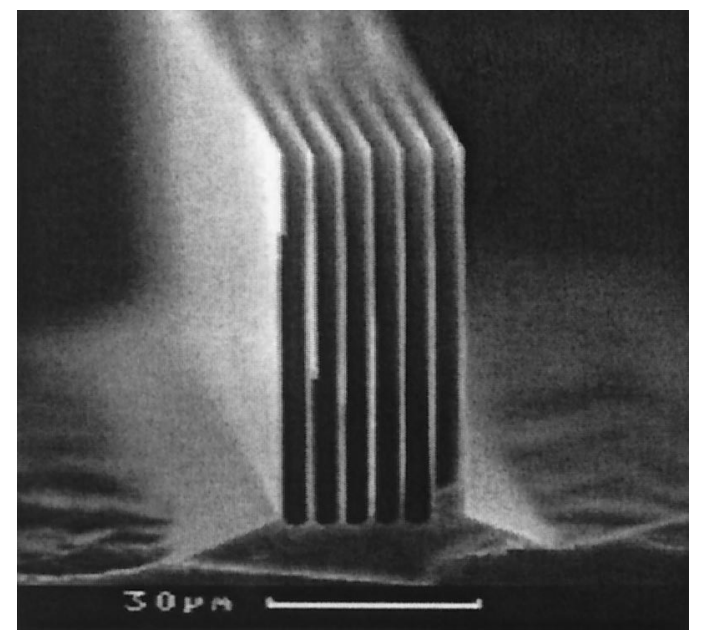

Fig. 1. Scanning electron microscopy image of $1 \mathrm{D}$ PC based on the periodically grooved $\mathrm{Si}$ with the lattice constant $A=4 \mu \mathrm{m}$, the number of lattice periods $m=7$, and the width of Si walls $D_{\mathrm{Si}}=0.8$ $\mu \mathrm{m}$.

minimal incident beam aperture of $10 \mu \mathrm{m} \times 20 \mu \mathrm{m} .{ }^{8}$ Samples they investigated had lattice constants of $A=16,8$, and $4 \mu \mathrm{m} .^{8,9} \quad$ For the sample with $A=4$ $\mu \mathrm{m}$, the main PBG can be partially observed in the range 10-15 $\mu \mathrm{m}$, whereas for the samples with $A=$ 16 and $8 \mu \mathrm{m}$, the PBG was out of the range under investigation. However, because of the high dielectric contrast $\left(n_{\mathrm{Si}} / n_{\text {air }}=3.42 / 1\right)$ of these structures, secondary stop bands with a high reflectance $R$ can be observed. Tolmachev et al.8,9 performed a fit between the calculated and the measured spectra of the secondary bandgaps as well as the main one, where available. This allowed a precise determination of the PC parameters $\left(D_{\mathrm{Si}}\right.$ and $\left.D_{\mathrm{Air}}\right)$. A precise knowledge of these values is obviously necessary for further calculations when this grooved Si structure will be used as a matrix for a composite PC. Moreover, the wide secondary bandgaps obtained ${ }^{8,9}$ are of particular interest since they allow the use of an additional shorter wavelength range without changing the lattice constant $A$. These results indicate the possibility of fabricating 1D PCs based on periodically grooved Si for the technologically important mid-IR range.

A considerable advantage of these 1D PCs based on grooved $\mathrm{Si}$ is that the air space can be infiltrated with a substance whose refractive index $n_{\text {space }}$ is different from $n_{\text {air }}$. This difference will obviously change the optical properties of the composite material. Therefore, by using periodically grooved $\mathrm{Si}$, we can either produce a family of $1 \mathrm{D}$ PCs or alternatively, we can build 1D composite PCs (CPCs). For example, in research by Tolmachev et al.,$^{9}$ a commercial nematic liquid crystal E7 with birefringence in the IR range of $\Delta n=\sim 0.2^{10}$ was infiltrated into a grooved Si matrix, leading to a change in the reflectance and to the manifestation of anisotropic properties in the composite structure. Even at this early stage of devel-

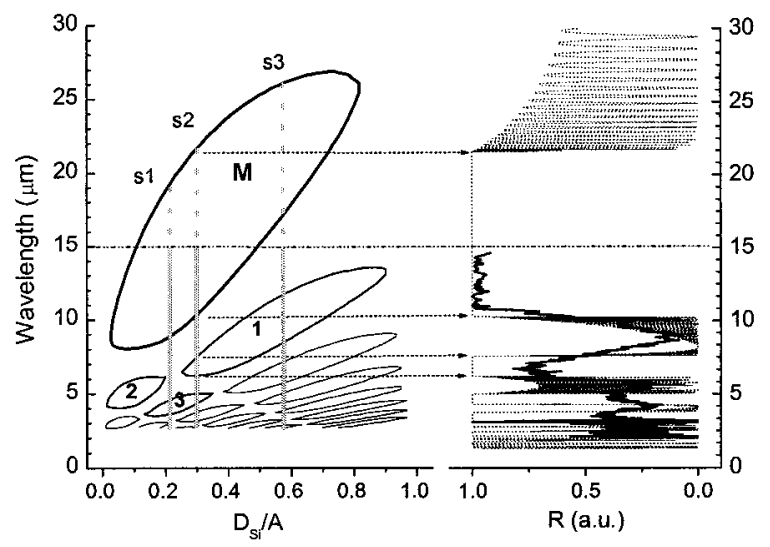

(a)

(b)

Fig. 2. (a) Calculations of the main PBG (M) and secondary (1, 2, 3 etc.) PBGs for a 1D PC based on the grooved Si with number of periods $m=7, A=4 \mu \mathrm{m}$ at normal incidence of light. (b) Calculated (dotted line) and measured (solid line) IR reflectance spectrum of sample S2. Note that the reflection band cannot be measured above $15 \mu \mathrm{m}$ owing to the cutoff of the detector.

opment of these 1D PCs the necessity for extraction of both optical and geometric design criteria, the socalled "gap map,"1 for these PC structures becomes obvious.

The objective in performing this research is to calculate the PBG regions for grooved Si structures acting as $1 \mathrm{D} \mathrm{PCs}$ and to compare these with those extracted from Fourier transform IR (FTIR) experimental data. This calculation and comparison allow us to determine the design criteria for these 1D PCs as a function of the fraction and the refractive index of one of the components and the number of structure periods, $m$.

\section{Calculations and Measurements of Photonic Bandgap Regions for a One-Dimensional Photonic Crystal Based on Grooved Si}

The characteristic matrix method ${ }^{11}$ was used to calculate the reflection spectra of a multilayered periodical structure. The following parameters were used during the calculations: normal incidence of light, number of periods in this structure $(m=7), n_{\mathrm{Si}}=$ 3.42 , and $n_{\text {air }}=1$. A scanning electron microscopy image of this structure is shown in Fig. 1.

For the calculations, the width of Si walls, $D_{\mathrm{Si}}$, is varied from $0.05 \mu \mathrm{m}$ up to a maximum value of 3.8 $\mu \mathrm{m}$ in $0.05-\mu \mathrm{m}$ steps. The lattice constant $A=$ $D_{\mathrm{Si}}+D_{\text {Air }}$, where $D_{\text {Air }}$ is the width of the air space, was taken as $4 \mu \mathrm{m}$ throughout. For each value of $D_{\mathrm{Si}} / A$, the fraction of the Si component in the periodic structure, the spectrum $R$ was calculated for wavelengths $\lambda$ between 2.5 and $30 \mu \mathrm{m}$. From each of the calculated spectra, the values of $\lambda$ with $R>99 \%$ were plotted against $\left(D_{\mathrm{Si}} / A\right)$ as shown in Fig. 2(a). Since the main parameter of interest is the wavelength range over which the $\mathrm{PC}$ will operate, we plot operating wavelength $\lambda$ versus $D_{\mathrm{Si}} / A$, allowing us to obtain the so-called "gap map"1 for the grooved Si PC. 
The closed area represents the PBG region. As expected, the main PBG, marked M, consists of an ellipse-shaped area for $D_{\mathrm{Si}} / A=0.03$ to 0.8. In addition, we also obtain a family of secondary PBGs (marked as 1,2, and 3), which are also ellipses but are much smaller than the main PBG. Note that the numbers on the corresponding secondary bandgaps are arbitrary. The reflection spectra for samples of grooved $\mathrm{Si}$ with the same lattice constant $A$ and $D_{\mathrm{Si}}=$ $0.8 \mu \mathrm{m}$ (sample S1) and $D_{\mathrm{Si}}=1.2 \mu \mathrm{m}$ (S2) were obtained from Refs. 8 and 9 whereas sample S3 with $D_{\mathrm{Si}}=2.3 \mu \mathrm{m}$ was measured in this study. Regions with maximum reflectance were determined with IR spectra from these samples. The results are plotted in Fig. 2(a) for values of $D_{\mathrm{Si}} / A=0.2,0.3$, and 0.575 together with the calculated data. The calculated data are given as a dashed line, whereas the experimental data are given as a thick solid line, which intersects the closed areas on Fig. 2(a). All data above the line at $15 \mu \mathrm{m}$ (dashed-dotted line) is calculated only because it cannot be measured since the cutoff of our UMA 500 MCT detector is at $\lambda>15$ $\mu \mathrm{m}$. Note that the calculated (dotted line) and the measured (solid line) IR reflectance spectrum of sample S2 is also shown in Fig. 2(b). In Fig. 2, we have explicitly illustrated the correspondence between the calculated and the experimental data for sample S2 for both the main and the first secondary gaps. It should be obvious from Fig. 2 that this agreement extends to other secondary gaps as well, but the lines showing this were omitted for clarity.

The spectral edges of these bands exactly coincide with the intersection points of the line corresponding to $D_{\mathrm{Si}} / A$ and the boundary line of the PBG regions. For sample S3 the main PBG is out of the range of our FTIR measurements owing to the cutoff of the detector as discussed earlier in this section. So, for this particular sample S3, only the calculated data for the main bandgap are presented with a dotted line, whereas for the secondary bandgaps both experimental and calculated data are shown with a solid line that intersects the PBG areas. Note that the experimental and the calculated data coincide well as was in the case of sample S2.

Using Fig. 2(a) it is possible now to choose the necessary parameters for the design of 1D PCs based on grooved $\mathrm{Si}$. As was shown in our investigations, ${ }^{8}$ the secondary bandgaps observed from grooved $\mathrm{Si}$ are also quite efficient. We may now predict the design criteria for using these bandgaps as stop bands suitable for different regions of IR spectra. In particular, using the gap map shown in Fig. 2, we can identify the range of wavelengths that can be practically realized for these structures. Consider the main PBG and the first three secondary PBGs. Starting from the fixed parameter $D_{\mathrm{Si}}$, we can predict the wavelength range in which this particular $\mathrm{PC}$ will work. Alternatively, we can determine the parameter $D_{\mathrm{Si}}$, which is necessary to obtain a PC over a certain wavelength range.

For example, if we want to use a $\mathrm{PC}$ with $D_{\mathrm{Si}} / A=$ 0.5 , we can determine from Fig. 2 that the wave-

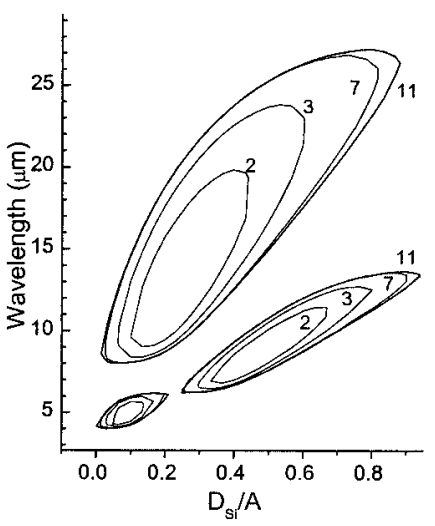

(a)

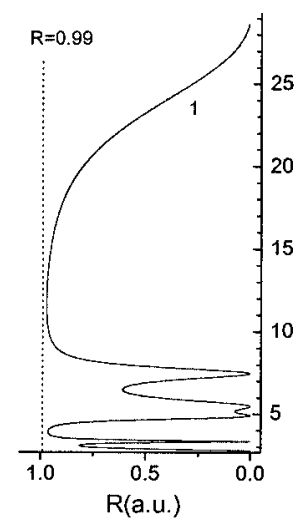

(b)
Fig. 3. (a) PBG regions for the main and the two closest secondary bandgaps versus filling factor $D_{\mathrm{Si}} / A$ and number of periods $m$ shown beside the respective bandgap. (b) Calculated reflectance spectrum for the grooved $\mathrm{Si}$ structure with 1 lattice period $(m=1)$. The lattice constant $A=4 \mu \mathrm{m}$ was used for all calculations.

length range of the main bandgap is between $\lambda=15$ and $25 \mu \mathrm{m}$. Alternatively, if we specify that we wish the wavelength range to have a maximum width between $\lambda=8$ and $11 \mu \mathrm{m}$, then we would need to have a $D_{\mathrm{Si}} / A=0.1$ if we wish to use the main gap. Alternatively, we can use the secondary PBG and a value of $D_{\mathrm{Si}} / A=0.53$.

Note that deep anisotropic etching of Si can reduce the width of the $\mathrm{Si}$ walls. ${ }^{7,8}$ The mechanism of grooves formation in $\mathrm{Si}$ is based on the difference in etching rate, $V$, for different crystallographic orientations, $\langle 110\rangle$ and $\langle 111\rangle$. As was shown by Kendall, ${ }^{7}$ a groove depth, $h$, up to $250 \mu \mathrm{m}$ can be obtained with minimal side etching of Si walls owing to the large ratio of $V_{\langle 110\rangle} / V_{\langle 111\rangle}=150-200$. Unfortunately, side etching of the $\mathrm{Si}$ walls increases with an increase in groove depth. This effect can play a crucial role for $D_{\mathrm{Si}}<1 \mu \mathrm{m}$. Obviously this effect must be reduced as much as possible. Thin Si walls may stick together and can even be destroyed if the wall depth is large. ${ }^{8}$ In practice, we choose for the purpose of our calculations a Si wall width larger than $0.3 \mu \mathrm{m}$. It is also necessary to take into account that the depth of Si walls in a structure must be large enough to allow the light beam to pass if IR measurements are necessary during the structure analysis. The minimal aperture of a standard FTIR microscope is $\sim 10 \mu \mathrm{m} \times$ $10 \mu \mathrm{m}$, and therefore the groove depth must be larger than $20 \mu \mathrm{m}$ in this particular case. However, for practical applications of $1 \mathrm{D}$ PC when the aperture of the light beam can be reduced to the size of the diameter of the optical fiber, a much smaller depth $(\sim 2-4 \mu \mathrm{m})$ can be used.

Furthermore, calculations of the PBG regions versus the number of the lattice periods $(m)$ from 2 to 11 have been performed with the same $A=4 \mu \mathrm{m}$ [see Fig. 3(a)]. The reflectance spectrum for $D_{\mathrm{Si}} / A=0.2$ (at $m=1$ and $D_{\mathrm{Si}}=0.6 \mu \mathrm{m}$ ) is shown in Fig. 3(b) as an example. As can be seen from this figure, the bands at $\approx 3$ and $10 \mu \mathrm{m}$ do not reach a value of $99 \%$ 


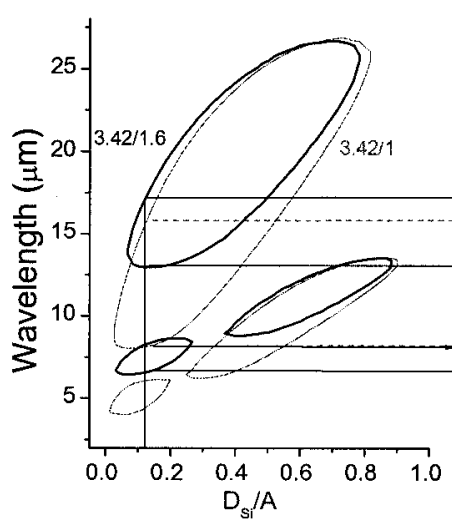

(a)

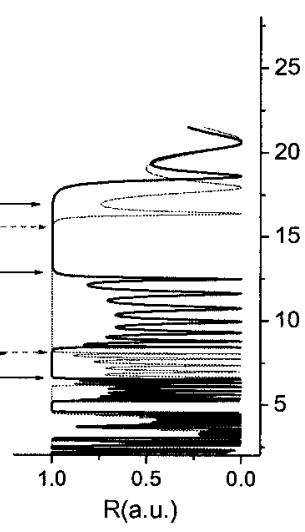

(b)
Fig. 4. (a) PBG regions for the main and the two closest secondary bandgaps for the empty $\left(n_{\mathrm{Si}} / n_{\text {space }}=3.42 / 1\right)$ and composite $\left(n_{\mathrm{Si}} /\right.$ $\left.n_{\text {space }}=3.42 / 1.6\right)$ grooved Si structures. (b) Calculated reflectance spectra for the same conditions with $D_{\mathrm{Si}} / A=0.125, A=4$ $\mu \mathrm{m}$, and $m=7$.

at maximum reflectance. We conclude that for this particular example the band map cannot be built up, since this method is based on the use of the spectral regions with $R>99 \%$. None of the calculated spectra obtained for $m=1$ and $D_{\mathrm{Si}}$ ranging from 0.1 up to $2.9 \mu \mathrm{m}$ have an $R$ value greater than $99 \%$. Therefore, to obtain the PBG it is not sufficient to fabricate a structure with one lattice period (two Si walls and one air space). Although, as can be seen in Fig. 3(b) these types of structure can have other uses, for example, as a mirror with a reflection in the range of $80 \%-97 \%$ if necessary. Calculations show that the reflection band in this case will be quite wide (from 9 up to $15 \mu \mathrm{m})$.

Increasing $m$ to 2 periods a structure with three $\mathrm{Si}$ walls and two air spaces leads to the appearance of ellipselike regions for both the main and the secondary gap maps as expected [Fig. 3(a)]. Further increases in $m$ from 3 to 11 periods result in an expansion of the PBG regions with practically no difference between PBG regions for $m=7$ and $m=11$. Therefore, when designing periodically grooved $\mathrm{Si}$ structures, with a filling factor $D_{\mathrm{Si}} / A$ variation in the range of $0.1-0.7$, there is no reason to fabricate more than 7 lattice periods, since further increase in $m$ do not result in any change in the optical characteristics of these structures.

A considerable advantage of $1 \mathrm{D}$ PCs based on grooved $\mathrm{Si}$ is that the air space can be infiltrated with a substance with a refractive index $n_{\text {space }}$ different from $n_{\text {air }}$. For example, as was shown by Tolmachev et al., ${ }^{8}$ grooves can be easily filled with liquid crystals. To understand the changes in the optical characteristics of composite structures, we performed a simulation of PBG regions using the average refractive index of liquid crystal in the isotropic phase, i.e., $n_{\text {space }}=1.6$. The PBG regions for the main and the two closest secondary bandgaps, obtained as a result of these simulations, are shown in Fig. 4(a) by the thick solid line. The PBG regions for the initial structure (with $n_{\text {space }}=1$ ) are also shown in Fig. 4 by a thin solid line for comparison. As can be seen from this figure the increase in the refractive index of the grooves from $n_{\text {space }}=1$ to $n_{\text {space }}=1.6$ leads to a decrease and shift of the PBG area. The important outcome is that filling the grooved structure with liquid crystal, with $D_{\mathrm{Si}} / A$ between 0.1 and 0.7 , results in the short wavelength edge of the main PBG being shifted substantially, whereas the long wavelength edge changes only a little. For $D_{\mathrm{Si}} / A \approx 0.6$, this edge remains practically unchanged. Therefore, when designing a composite structure, one must bear in mind that the spectra of the initially empty periodically grooved Si structure and that after the air gaps have been filled with liquid crystals can be very different. An example is shown in Fig. 4(b) in which the secondary bandgap shown at $5 \mu \mathrm{m}$ in the empty structure completely disappears after filling the grooves with a substance with a refractive index of 1.6. However, a bandgap at $7.5 \mu \mathrm{m}$ does appear instead. The authors are working on a more detailed investigation of the composite structures based on periodically grooved $\mathrm{Si}$ infiltrated with liquid crystals and will publish their findings in a forthcoming paper.

\section{Conclusions}

Calculations of the PBG regions for periodically grooved silicon acting as 1D PC have been performed. On the basis of these calculations a gap map was produced for a PC based on grooved $\mathrm{Si}$ with a lattice constant $A=4 \mu \mathrm{m}$ and with various $\mathrm{Si}$ wall widths $D_{\mathrm{Si}}$, a number of lattice periods, and a groove refractive index. This gap map allows the design of $1 \mathrm{D}$ PCs with gaps in user-specified wavelength ranges simply by altering the geometry. Alternatively, we can use this map to predict the wavelength range over which a particular PC will perform. FTIR measurements of the PBGs performed for a number of grooved $\mathrm{Si}$ samples in the range $2.5-15 \mu \mathrm{m}$ show excellent agreement with the calculated PBGs.

The authors thank the Enterprise Ireland International Programme (IC/2002/029), the European Commission (INTAS 01-0642), and the Russian government contract N40.072.1.1.1180 for partial support of this research. In addition, we thank E. Astrova and S. Gaponenko for privately discussing this research with us.

\section{References}

1. J. D. Joannopoulos, R. D. Meade, and J. N. Winn, Photonic Crystals: Molding the Flow of Light (Princeton University Press, Singapore, 1995).

2. Y. Fink, J. N. Winn, F. Shanhui, C. Chiping, J. Michel, J. D. Joannopoulos, and E. L. Thomas, "A dielectric omnidirectional reflector," Science 282, 1679-1682 (1998).

3. D. N. Chigrin, A. V. Lavrinenko, D. A. Yarotsky, and S. V. Gaponenko, "All-dielectric one-dimensional periodic structures for total omnidirectional reflection and partial spontaneous emission control," J. Lightwave Technol. 17, 2018-2024 (1999).

4. J. S. Foresi, P. R. Villeneuve, J. Ferrera, E. R. Thoen, G. Steinmeyer, S. Fan, J. D. Joannopoulos, L. C. Kimerling, H. I. 
Smith, and E. P. Ippen, "Photonic-bandgap microcavities in optical waveguides," Nature 390, 143-513 (1997).

5. P. R. Villeneuve, S. Fan, J. D. Joannopoulos, Lim Kuo-Yi, G. S. Petrich, L. A. Kolodziejski, and R. Reif, "Air-bridge microcavities," Appl. Phys. Lett. 67, 167-169 (1995).

6. V. A. Tolmachev, L. S. Granitsyna, E. N. Vlasova, B. Z. Volchek, A. V. Nashchekin, A. D. Remenyuk, and E. V. Astrova, "One-dimensional photonic crystal obtained by vertical anisotropic etching of silicon," Semiconductors 36, 996-1000 (2002).

7. D. L. Kendall, "Vertical etching of silicon at very high aspect ratios," Ann. Rev. Mater. Sci. 9, 373-403 (1979).
8. V. Tolmachev, T. Perova, E. Astrova, B. Volchek, and J. K. Vij, "Vertically etched silicon as 1D photonic crystal," Phys. Status Solidi A 197, 544-548 (2003).

9. V. A. Tolmachev, T. S. Perova, J. K. Vij, E. V. Astrova, K. Berwick, and A. Moore, "FTIR and Raman investigation of vertically etched silicon as $1 \mathrm{D}$ photonic crystal," in OptoIreland 2002: Optics and Photonics Technologies and Applications, T. G. Glynn, ed., Proc. SPIE 4876, 196-205 (2003).

10. S.-T. Wu, "Infrared properties of nematic liquid crystal: an overview," Opt. Eng. 26, 120-128 (1987).

11. R. M. A. Azzam and N. M. Bashara, Ellipsometry and Polarized Light (North-Holland, Amsterdam, Netherlands, 1977). 